\title{
Solution of the time dependent Dirac-Fock-Slater equation for many-electron collisions systems using a time window method is
}

\author{
P. Kürpick ${ }^{\text {a,* }}$, W.-D. Sepp ${ }^{\text {a }}$, H.J. Lüdde ${ }^{\text {b }}$, B. Fricke ${ }^{\text {a }}$ \\ ${ }^{a}$ Fachbereich Physik, Universität Kassel, 34109 Kassel, Germany \\ ${ }^{\mathrm{b}}$ Institut für theoretische Physik, Universität Frankfurt, 60486 Frankfurt, Germany
}

Received 13 June 1994

\begin{abstract}
We present a new scheme to solve the time dependent Dirac-Fock-Slater equation (TDDFS) for heavy many electron ion-atom collision systems. Up to now time independent self consistent molecular orbitals have been used to expand the time dependent wavefunction and rather complicated potential coupling matrix elements have been neglected. Our idea is to minimize the potential coupling by using the time dependent electronic density to generate molecular basis functions. We present the first results for $16 \mathrm{MeV} \mathrm{S}^{16+}$ on Ar.
\end{abstract}

We take up the problem of solving the time dependent Dirac-Fock (TDDF) equation for many-electron collision systems. Starting from the independent particle model we have to solve an effective time dependent single-particle equation

$$
\begin{aligned}
& (\underbrace{\left(\hat{h}_{\mathrm{e}}+V^{\mathrm{C}}(\rho(r, t))+V^{\mathrm{Ex}}(\rho(r, t))\right.}_{\hat{h}^{\mathrm{TDDFS}}}-\mathrm{i} \hbar \frac{\partial}{\partial t}) \\
& \quad \times \psi_{l}(r, t)=0
\end{aligned}
$$

for all $N$ electrons involved in the collision system. $V^{\mathrm{C}}$ and $V^{\mathrm{Ex}}$ are the Coulomb and exchange potentials as functions of the time dependent charge density during the collision and $\hat{h}_{\mathrm{el}}$ is the single-particle Hamiltonian

$\hat{h}_{\mathrm{el}}=c \alpha p+(\beta-1) m c^{2}$.

In the close coupling approximation applied by us up to now a predefined self consistent solution of the static Dirac-Fock equation

$h^{\mathrm{SCF}} \phi_{k}^{\mathrm{SCF}}(\boldsymbol{r}, \boldsymbol{R})=\epsilon_{k} \phi_{k}^{\mathrm{SCF}}(\boldsymbol{r}, \boldsymbol{R})$

was used to describe the time dependent wavefunctions

$\psi_{l}(r, t)=\sum_{k}^{M} a_{k l}(t) \phi_{k}^{\mathrm{SCF}}(\boldsymbol{r}, \boldsymbol{R})$.

for all internuclear distances $R$ [1].

\footnotetext{
This paper was through a mistake not included in the Proceedings of the 5th Workshop on Fast Ion-Atom Collision Processes, Nucl. Instr. and Meth. B $86(1,2)(1994)$.

${ }^{*}$ Corresponding author.
}

This ansatz leads to the usual close coupling equations

$$
\begin{aligned}
\dot{a}_{m l}(t)= & \sum_{k}^{M} a_{k l}(t)\left\langle\phi_{m}^{\mathrm{SCF}}(r, R)\right. \\
& \left.\times|\underbrace{\hat{h}^{\mathrm{TDDFS}}-\hat{h}^{\mathrm{SCF}}}_{\Delta \hat{h}^{\mathrm{Pot}}}+\hat{h}^{\mathrm{SCF}}-\mathrm{i} \hbar \frac{\partial}{\partial t}| \phi_{k}^{\mathrm{SCF}}(\boldsymbol{r}, \boldsymbol{R})\right\rangle
\end{aligned}
$$

where the potential matrix elements $\Delta \hat{h}^{\text {Pot }}$ are rather complicated to evaluate and have therefore been neglected up to now. Asymptotically $\Delta \hat{h}^{\mathrm{Pot}}=0$ but during the collision the dynamic electronic rearrangements via excitation and charge transfer produces significant changes from the static self consistent charge distribution. The idea is to include parts of the time dependent potential matrix elements $\Delta \hat{h}^{\text {Pot }}$ by dividing the integration interval of the variable $t$ into windows. Instead of using the self consistent energy eigenvalues and matrix elements for all $-\infty \leq t \leq+\infty$ we readjust the molecular basis at each window boundary.

Within a window we expand the time dependent wavefunction in molecular basis functions which we get from solving the static Dirac-Fock equation

$$
\begin{gathered}
\hat{h}^{\mathrm{MO}}\left(\tilde{\rho}\left(r, t_{i}, \boldsymbol{R}(t)\right)\right) \phi_{k}^{(i)}\left(r, t_{i}, \boldsymbol{R}(t)\right) \\
=\epsilon_{k}\left(t_{i}, \boldsymbol{R}(t)\right) \phi_{k}^{(i)}\left(r, t_{i}, \boldsymbol{R}(t)\right) .
\end{gathered}
$$

using the time dependent electronic density

$$
\begin{aligned}
\tilde{\rho}\left(\boldsymbol{r}, t_{i}, \boldsymbol{R}(t)\right)= & \sum_{l}^{N} \sum_{k}^{M} \sum_{m}^{M} a_{k l}^{(i-1)^{+}}\left(t_{i}\right) a_{m l}^{(i-1)}\left(t_{i}\right) \phi_{k}^{(i-1)^{+}} \\
& \times\left(\boldsymbol{r}, t_{i}, \boldsymbol{R}(t)\right) \phi_{m}^{(i-1)}\left(\boldsymbol{r}, t_{i}, \boldsymbol{R}(t)\right)
\end{aligned}
$$

from the beginning of the time window being decomposed via a Mulliken analysis, where 
- $a_{m l}^{(i-1)}\left(t_{i}\right)$ stands for the time dependent single-particle amplitudes in the $(i-1)$ th time window at the time $t_{i}$ and

- $\phi_{m}^{(i-1)}\left(r, t_{i}, R(t)\right)$ stands for the molecular basis functions in the $(i-1)$ th time window at the time $t_{i}$.

For $t$ within the $i$ th time window the molecular wavefunctions $\phi_{k}^{(i)}\left(r, t_{i}, \boldsymbol{R}(t)\right)$ are used as a basis set to expand the time dependent single-particle wavefunction $\psi_{l}(r, t)$

$\psi_{l}(r, t)=\sum_{k}^{M} a_{k l}^{(i)}(t) \phi_{k}^{(i)}\left(r, t_{i}, \boldsymbol{R}(t)\right)$.

At the beginning of each time window we project the time dependent wavefunctions $\psi_{i}(r, t)$ on the new basis $\phi_{k}^{(i+1)}\left(r, t_{i}, R(t)\right)$. The new time dependent single-particle amplitudes $a_{k l}^{(i+1)}\left(t_{i}\right)$ are therefore given by

$$
\begin{aligned}
a_{k l}^{(i+1)}\left(t_{i}\right)= & \sum_{l}^{M} a_{m l}^{(i)}\left(t_{i}\right)\left\langle\phi_{k}^{(i+1)}\left(r, t_{i}, R(t)\right)\right. \\
& \left|\phi_{m}^{(i)}\left(\boldsymbol{r}, t_{i}, \boldsymbol{R}(t)\right)\right\rangle,
\end{aligned}
$$

for $k=1, \ldots, M$ and $l=1, \ldots, N$.

The integration of the close coupling equations

$$
\begin{aligned}
\dot{a}_{m l}^{(i)}(t)= & \sum_{k}^{M} a_{k l}^{(i)}(t)\left\langle\phi_{m}^{(i)}\left(r, t_{i}, R(t)\right)\right. \\
& \times\left|\dot{R} \frac{\partial}{\partial R}-\frac{\mathrm{i}}{\hbar} L_{y}+\epsilon_{k}^{(i)}\left(t_{i}, R(t)\right)\right| \\
& \left.\times \phi_{k}^{(i)}\left(r, t_{i}, R(t)\right)\right\rangle
\end{aligned}
$$

is then done for $l=1, \ldots, N$ and $t_{i} \leq t \leq t_{i+1}$.

The time dependent single-particle equation has to be solved separately for each impact parameter and collision energy.

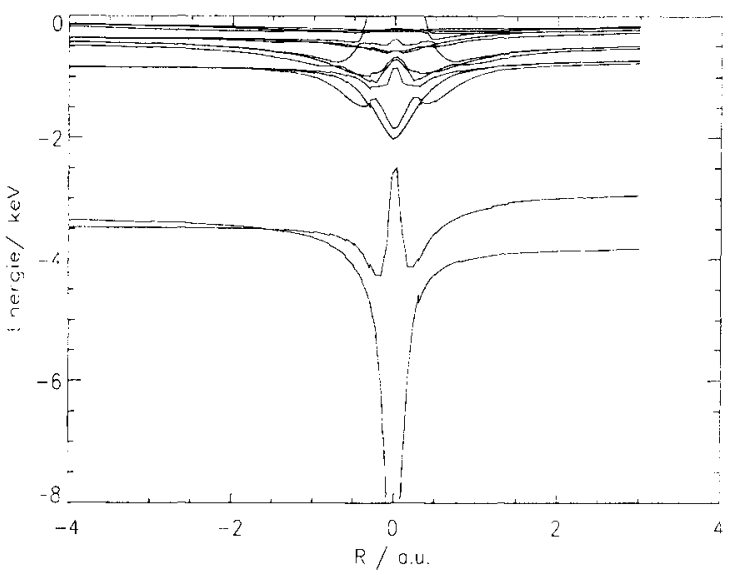

Fig. 1. Correlation diagram for $16 \mathrm{MeV} \mathrm{S}^{16+}$ on $\mathrm{Ar}$ at an impact parameter of 0.0274 a.u. Negative $R$ values stand for the incoming part of the trajectory and positive $R$ values for the outgoing part of the trajectory.
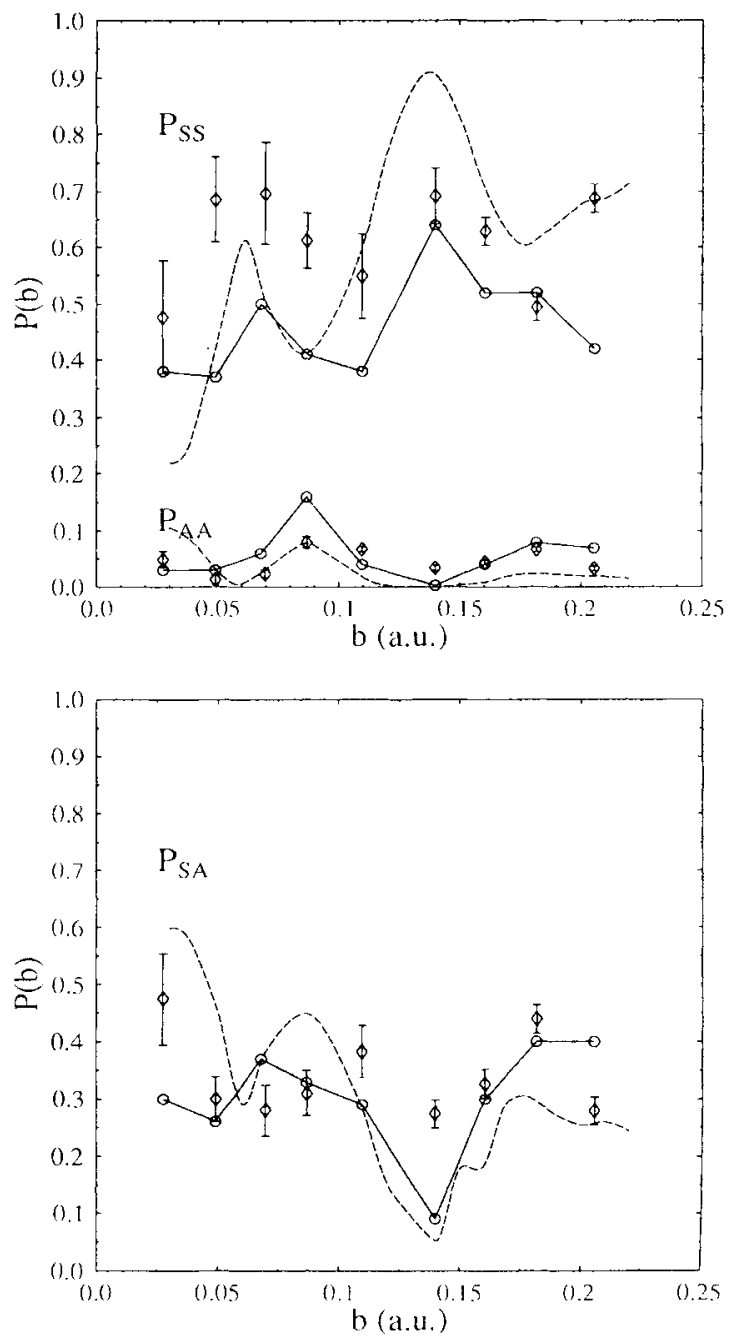

Fig. 2. Upper figure: $P_{\mathrm{SS}}$ and $P_{\mathrm{AA}}$, lower figure: $P_{\mathrm{SA}}$. Experimental values: Schulz et al. [2]; full curve: our TDDFS results; broken curve: our SCF results [3].

We present the first results for the collision system 16 $\mathrm{MeV} \mathrm{S}^{16+}$ on Ar where detailed triple coincidence measurements between two $\mathrm{K}_{\alpha} \mathrm{X}$-rays and an angle resolved projectile are available from Schulz et al. $[2,3]$. The measurements provide the probabilities $P_{\mathrm{SS}}, P_{\mathrm{AA}}$ and $P_{\mathrm{SA}}$, which give

- the probability to find two holes in the S K-shell $\left(P_{\mathrm{SS}}\right)$,

- to find two holes in $\operatorname{Ar} \mathrm{K}$-shell $\left(P_{\mathrm{AA}}\right)$, and

- to find one hole in the Ar K-shell and one hole in the S K-shell.

The correlation diagram for the innermost impact parameter of $b=0.0274$ a.u. is shown in Fig. 1. A strong asymmetry between the incoming and outgoing trajectory can be seen both in the $\mathrm{K}$-shells and the higher lying $\mathrm{L}$ - 
and M-shells. Negative $R$ values stand for the incoming trajectory and positive $R$ values for the outgoing trajectory. Due to the finite size of the time window we get small discontinuities in the molecular eigenvalues at the window boundaries. The size of the windows have been chosen according to the maxima in the dynamic coupling matrix elements we got from a former SCF calculation. Therefore in the asymptotic region large windows took place while the size of the windows was chosen to be small around the point of closest approach.

Fig. 2 shows the experimental values for the probabilities $P_{\mathrm{SS}}, P_{\mathrm{AA}}$ and $P_{\mathrm{SA}}$ versus our results calculated from the single-particle amplitudes using the inclusive probability formalism $[4,5]$ and presented as full curves. For $P_{\mathrm{SS}}$ and $P_{\mathrm{AA}}$ a good agreement with the oscillatory structure of the experimental values is reached. We slightly underestimate the elastic channel $P_{\mathrm{SS}}$ for small impact parameters. The broken curves in Fig. 2 show the inclusive probabilities for $P_{\mathrm{SS}}, P_{\mathrm{AA}}$ and $P_{\mathrm{SA}}$ from a former SCF calculation. Comparing the SCF results with the TDDFS results the later have a less pronounced oscillatory structure which fits better with the experimental results.
Finally the remaining discrepancy between both the SCF and the TDDFS results and the experimental results may even be correct because the experimental probabilities $P_{\mathrm{SS}}, P_{\mathrm{AA}}$ and $P_{\mathrm{SA}}$ were calculated supposing the relation $P_{\mathrm{SS}}+P_{\mathrm{AA}}+P_{\mathrm{SA}}=1$

to be valid for all impact parameters [2]. This is probably not true for small impact parameters where other inelastic channels become dominant.

\section{References}

[1] W.-D. Sepp, D. Kolb, W. Sengler, H. Hartung, B. Fricke, Phys. Rev. A 33 (1986) 3679.

[2] M. Schulz, E. Justiniano, J. Konrad, R. Schuch, A. Salin, J. Phys. B 20 (1987) 2057.

[3] P. Kürpick, B. Thies, W.-D. Sepp, B. Fricke, J. Phys. B 24 (1991) L139.

[4] P. Kürpick, H.J. Lüdde, Comp. Phys. Comm. 75 (1993) 127.

[5] P. Kürpick, Ph.D. thesis, University of Kassel (1993). 